\title{
Effects of a synbiotic containing Lactobacillus acidophilus ATCC 4962 on plasma lipid profiles and morphology of erythrocytes in hypercholesterolaemic pigs on high- and low-fat diets
}

\author{
Min-Tze Liong ${ }^{1 *}$, Frank R. Dunshea ${ }^{2}$ and Nagendra P. Shah ${ }^{3}$ \\ ${ }^{1}$ School of Industrial Technology, Food Technology Division, University of Science Malaysia, 11800 Minden, Penang, Malaysia \\ ${ }^{2}$ Faculty of Land and Food Resources, The University of Melbourne, Parkville, Victoria 3010, Australia \\ ${ }^{3}$ School of Molecular Sciences, Victoria University, Werribee Campus, PO Box 14428, Melbourne, Victoria 8001, Australia
}

(Received 20 December 2006 - Revised 28 February 2007 - Accepted 12 March 2007)

\begin{abstract}
The aim of this study was to evaluate the effect of a synbiotic containing Lactobacillus acidophilus ATCC 4962, fructooligosaccharide, inulin and mannitol on plasma lipid profiles and erythrocyte membrane properties in hypercholesterolaemic pigs on high- and low-fat diets. Twenty-four white male Landrace pigs were randomly allocated to four treatment groups for 8 weeks $(n 6)$. Treatment factors were the supplementation of synbiotic (with and without) and dietary fat (5 and $15 \%)$. The supplementation of synbiotic reduced plasma total cholesterol $(P=0.001)$, TAG $(P=0.002)$ and LDL-cholesterol $(P=0.045)$ for both dietary fats. A higher concentration of esterified-cholesterol in HDL of pigs supplemented with synbiotic than the control regardless of dietary fat $(P=0.036)$ indicated that cholesterol was reduced in the form of cholesteryl esters. Reduced concentration of cholesteryl esters $(P<0.001)$ and increased concentration of TAG $(P=0.042)$ in LDL of pigs on synbiotic suggested that LDL-cholesterol was reduced via the hydrolysis of smaller and denser LDL particles. The erythrocytes of pigs without any synbiotic showed more prevalence of spur cells than those given the synbiotic, as supported by the higher cholesterol: phospholipid ratio in erythrocytes $(P=0.001)$. Also, membrane fluidity and rigidity were improved as supported by the decreased fluorescence anisotropies in the Hb-free erythrocyte membrane of pigs given synbiotic $(P<0.001)$. The administration of the synbiotic reduced plasma TAG, total cholesterol and LDL-cholesterol in hypercholesterolaemic pigs, possibly in the form of cholesteryl esters, via the interrelated pathways of lipid transporters (VLDL, LDL and HDL). The synbiotic also reduced deformation of erythrocytes via improved membrane fluidity and permeability.
\end{abstract}

Lactobacillus: Cholesterol: Fructooligosaccharide: Inulin: Mannitol

Probiotics are defined as 'live microorganisms that, when administered in adequate amounts, confer a health benefit on the host' ${ }^{1}$. Prebiotics are a more recent concept and are defined as chemical substances that act as substrates specifically for the host's intrinsic probiotic bacteria, and thus encourage their growth ${ }^{2}$. A combination of probiotic and prebiotic is termed synbiotic, which has been used to improve various aspects of human health ${ }^{3,4}$.

High-fat diets have been associated with hypercholesterolaemia as indicated by higher than normal serum total cholesterol $^{5}$. Studies have shown that $1 \mathrm{mmol}$ higher than normal cholesterol concentration could increase the risk of CHD by $35 \%$ and the risk of coronary death by $45 \%$, and a small reduction in the serum cholesterol of $1 \%$ could reduce the risk of CHD by $2-3 \%^{6}$. Numerous dietary approaches have been used to improve hypercholesterolaemia including the usage of synbiotics. Several possible mechanisms for cholesterol removal by probiotics and prebiotics have been proposed including assimilation of cholesterol by growing cells, binding of cholesterol to cellular surface, incorporation of cholesterol into the cellular membrane ${ }^{7}$, deconjugation of bile via bile salt hydrolase, co-precipitation of cholesterol with deconjugated bile $^{8}$, binding action of bile by fibre ${ }^{9}$ and production of SCFA by oligosaccharides ${ }^{10}$. Although numerous studies have shown promising results in reducing TAG, total cholesterol and LDL-cholesterol concentrations after consumption of probiotic and/or prebiotics ${ }^{11}$, their effects on serum lipids have been controversial with numerous studies indicating inconclusive results ${ }^{12}$. Also, most of these studies involved continuous feeding of high-cholesterol diets containing high concentrations of probiotics and/or prebiotics. In recent years, consumers are becoming nutritionally conscious, and adopt diets containing MUFA and PUFA, and minimize intake of total fat. Very little information is available on the cholesterol-lowering effect of synbiotic in hypercholesterolaemic subjects after cessation of high-fat diets.

A basic understanding of the metabolism of the plasma lipoproteins, and the major lipids that they transport is of primary importance. Because the metabolism of the plasma lipoproteins is highly interrelated, it is important to consider each

Abbreviations: ANS, 8-anilino-1-napthalenesulphonic acid; CE, cholesteryl esters; C/P, ratio of cholesterol/phospholipids; DPH, 1,6-diphenyl-1,3,5-hexatriene; FAn, fluorescence anisotropy; FOS, fructooligosaccharide; TMA-DPH, 1-(4-trimethylammonium)-6-phenyl-1,3,5-hexatriene.

* Corresponding author: Dr Liong Min Tze, fax +604 657 3678, email mintze.liong@usm.my 
of the lipoproteins and their properties instead of focusing only on the main lipid classes to better understand the complete lipid profile ${ }^{13}$.

When rabbits, guinea pigs and dogs were fed high-cholesterol diets, morphological defects of the erythrocytes including the formation of spur cells was observed ${ }^{14}$, where the enterocytes are covered with spike-like formations that may vary in width, length and distribution. Although the role of this abnormality remains unknown, the occurrence of spur cells contributes to the entrapment and destruction of erythrocytes in the spleen and has been reportedly caused by increased membrane rigidity ${ }^{14}$. Any changes in the erythrocyte membranes would involve modifications on the lipid order due to the fact that the erythrocyte membrane is an amphiphilic phospholipid layer containing protein and cholesterol. Fluorescence probes are used to evaluate modifications in the membrane packing order via incorporation into different sites in the bilayer, namely the apolar regions, interface between the polar head and apolar tail, and in the polar surface ${ }^{15}$.

We have previously screened and developed a synbiotic product consisting of Lactobacillus acidophilus ATCC 4962, mannitol, fructooligosaccharides (FOS) and inulin that specifically targeted removal of cholesterol in laboratory media containing cholesterol ${ }^{16}$. However, the effect of such synbiotic on plasma lipid profiles and erythrocytes in in vivo models has not been studied. Thus, the aims of the present study were to evaluate (1) the effect of such a synbiotic on plasma lipoproteins and the possible mechanisms via lipid transporters, and (2) the effect of the synbiotic on the morphology of erythrocytes and the possible mechanisms via alteration on membrane fluidity, by using pigs given high- and low-fat diets. The effect of the synbiotic during a continuum of the high-fat diet and after the cessation of the high-fat diet will also be evaluated.

\section{Material and methods}

\section{Source of probiotic culture and prebiotics}

L. acidophilus ATCC 4962 is a human-derived strain that was obtained from the Australian Starter Culture Research Center (ASRC; Werribee, Australia). The stock culture was stored in $40 \%(\mathrm{v} / \mathrm{v})$ glycerol at $-80^{\circ} \mathrm{C}$. The organism was subcultured three times in sterile de Mann, Rogosa, Sharpe (MRS) broth using $1 \%(\mathrm{v} / \mathrm{v})$ inoculum and $20 \mathrm{~h}$ of incubation at $37^{\circ} \mathrm{C}$ prior to use and was stored at $4^{\circ} \mathrm{C}$ between transfers. A freeze-dried culture (containing approximately $9.0 \log _{10}$ colony-forming units/g) was used in the present study. For freeze-drying, the cell pellet obtained from harvesting the fermentation broth was suspended in $0.1 \mathrm{M}$-phosphate buffer (pH 6.8) containing $2.0 \%(\mathrm{w} / \mathrm{v})$ of food grade cryoprotectant Unipectin $^{\text {TM }}$ RS 150 (Savannah Bio Systems, Balwyn East, Australia), frozen at $-20^{\circ} \mathrm{C}$ and freeze-dried (Dynavac FD300; Airvac Engineering Pty Ltd, Rowville, Australia) at $-20^{\circ} \mathrm{C}$ and $-100 \mathrm{kPa}$. Three commercially available prebiotics were used including mannitol (Mannogem; SPI Polyols Inc., New Castle, DE, USA), FOS (Raftilose P95; Orafti Pty Ltd, Tienen, Belgium) and inulin (Raftilene ST; Orafti). The FOS had a purity of $95 \%$ and the remaining $5 \%$ contained glucose, fructose and sucrose. The degree of polymerization of FOS ranged from 2 to 7, with an average of 4 . The inulin had a purity of $92 \%$, and an average degree of polymerization of 10. The mannitol had a degree of polymerization between 1 and 2 and was affirmed Generally Regarded As Safe (GRAS; Code of Federal Regulations, Title 21 CFR 180.25).

\section{Animals and diets}

Twenty-four crossbred (Large White $\times$ Landrace) pigs (initial weight 33 (SD $8 \mathrm{~kg}$ )) were used. The study was approved by the Animal Ethics Committee of the Department of Primary Industry (Werribee, Australia). Pigs were housed in individual pens in a randomized block design according to their initial live weight and four treatments. The animals were kept on a basal diet containing $15 \%$ fat for 2 weeks to induce hypercholesterolaemia. After this period, pigs were given either a high-fat (15\% fat) or a low-fat $(5 \%)$ diet for 8 weeks. The pigs on the synbiotic diet were supplemented with powdered L. acidophilus ATCC 4962 (1.00 g/pig per d), FOS ( $1.25 \mathrm{~g} /$ pig per d), mannitol ( $1.56 \mathrm{~g} /$ pig per d) and inulin $(2.20 \mathrm{~g} / \mathrm{pig}$ per $\mathrm{d})$, while pigs on the control were not supplemented with synbiotic. The composition of the synbiotic was based on our previous in vitro optimization study where inoculum size of $L$. acidophilus ATCC 4962, and concentrations of mannitol, FOS and inulin were the significant factors for optimum removal of cholesterol ${ }^{16}$. The compositions of the high- and low-fat diets are shown in Table 1. Pigs were kept in a room with controlled temperature $\left(20-22^{\circ} \mathrm{C}\right)$ and humidity $(50-55 \%)$ and maintained in a cycle of light for $12 \mathrm{~h}(06 \cdot 00-18.00$ hours) and dark for $12 \mathrm{~h}$ (18.00-06.00 hours). The body weight of each pig was recorded weekly and the amount of the basal diet adjusted individually according to their energy intake per weight; $16.9 \mathrm{MJ} / \mathrm{kg}$ and $14.6 \mathrm{MJ} / \mathrm{kg}$ for high-fat diet and low-fat diet, respectively. Pigs were fed on a daily basis and allowed to consume ad libitum water during the experimental period.

\section{Preparative procedures}

All pigs were fasted overnight prior to blood collection weekly. Immediately after bleeding, pigs were fed their respective diets and non-fasting blood was collected after $3 \mathrm{~h}$ of feeding. Fasting and non-fasting blood samples were obtained by venepuncture. Heparin was used as anticoagulant. All data are presented using fasted blood samples unless stated otherwise.

The whole blood was centrifuged for $20 \mathrm{~min}$ at $2714 \mathrm{~g}$ (Sorvall RT7; Newtown, CT, USA) to separate the plasma from the erythrocyte pellet. The plasma was recentrifuged for $30 \mathrm{~min}$ at $18879 \mathrm{~g}$ (Beckman Coulter, Fullerton, CA, USA) to remove chylomicrons.

A discontinuous density gradient ultracentrifugal procedure was used to fractionate plasma lipoproteins as previously described $^{17}$. Chylomicron-free plasma was adjusted with $\mathrm{NaCl}-\mathrm{KBr}$ solution $(1.346 \mathrm{~g} / \mathrm{ml})$ to desired densities of $1.006,1.063$ and $1.21 \mathrm{~g} / \mathrm{ml}^{18}$. Gradients were centrifuged at $40000 \mathrm{rpm}$ for $20 \mathrm{~h}$ at $15^{\circ} \mathrm{C}$ (Beckman model TI ultracentrifuge with type $70 \cdot 1$ rotor) and subfractionated by successive downwards aspiration using a Pasteur pipette. VLDL was isolated at density $<1.006 \mathrm{~g} / \mathrm{ml}$ and the infranet obtained was refloated at a density of $1.063 \mathrm{~g} / \mathrm{ml}$ to obtain LDL (density $1.006-1.063 \mathrm{~g} / \mathrm{ml})$. Similarly, HDL was obtained at density $1.063-1.21 \mathrm{~g} / \mathrm{ml}$. 
Table 1. Composition of the low-fat (5\% fat) and high-fat (15\% fat) basal diet $^{*}$

\begin{tabular}{lcc}
\hline Ingredient & $\begin{array}{c}\text { Low-fat diet } \\
\text { (\% dry mass) }\end{array}$ & $\begin{array}{c}\text { High-fat diet } \\
\text { (\% dry mass) }\end{array}$ \\
\hline Total fat & 5.00 & 15.00 \\
Wheat (11\% crude protein) & 78.40 & 61.64 \\
Soyabean meal (48\% crude protein) & 7.82 & 14.29 \\
Meat and bone meal & 7.50 & 7.50 \\
$\quad$ (50\% crude protein) & & \\
Blood meal (85\% crude protein) & 2.50 & 2.50 \\
Tallow & 1.60 & 8.40 \\
Sunflower oil & 0.80 & 4.20 \\
Dicalcium phosphate & 0.72 & 0.72 \\
Salt & 0.20 & 0.20 \\
Vitamin-mineral premix & 0.20 & 0.20 \\
Lysine & 0.15 & 0.15 \\
Methionine & 0.07 & 0.10 \\
Threonine & 0.00 & 0.05 \\
Tylan & 0.04 & 0.04 \\
Fatty acids (\% of total fatty acids) & & \\
SFA & 21.8 & 26.0 \\
MUFA & 27.3 & 29.3 \\
PUFA & 50.9 & 44.7 \\
\end{tabular}

* Feed was adjusted weekly according to energy intake per weight of pigs $14.6 \mathrm{MJ} / \mathrm{kg}$ for those on the basal diet containing $5 \%$ fat and $16.9 \mathrm{MJ} / \mathrm{kg}$ for those on the basal diet containing $15 \%$ fat.

For the preparation of $\mathrm{Hb}$-free erythrocyte membrane (erythrocyte ghosts), erythrocyte pellets were washed twice with saline to remove any remaining plasma and buffy coat, followed by lysing in 30 volumes of $5 \mathrm{mM}-\mathrm{Na}_{2} \mathrm{HPO}_{4}$ buffer, $\mathrm{pH} 8 \cdot 0^{19}$ and isolating by centrifugation. Erythrocyte ghosts were washed with the same buffer until $\mathrm{Hb}$-free.

\section{Analytical procedures}

Chylomicron-free plasma was analysed for total, HDLcholesterol, LDL-cholesterol and TAG using commercial kits (Thermo Electron Corp., Melbourne, Australia). Plasma subfractions of VLDL, LDL and HDL were analysed for TAG, protein, phospholipids, cholesteryl esters (CE) and free cholesterol concentrations. TAG and total cholesterol were analysed using commercial kits (Thermo Electron Corp.). CE were determined using the Amplex Red Reagent Kit (Molecular Probes, Eugene, OR, USA) and free cholesterol was determined as the difference between total cholesterol and $\mathrm{CE}^{20}$. The protein content was determined with bovine serum albumin as the standard ${ }^{21}$. A factor of 0.8 was used to convert bovine serum albumin protein into lipoprotein protein $^{22}$. The total phospholipid content was measured as phosphate as previously described ${ }^{23}$ and a factor of 25 was used for conversion into serum phospholipids ${ }^{14}$.

The erythrocyte count was carried out using a Newbauer haemocytometer. The morphology of erythrocytes was assessed using Wright's stain smears ${ }^{24}$. The erythrocyte total cholesterol and phospholipids were determined after lipid extraction with acetone-ethanol (1:1). The total cholesterol and phospholipids were measured as described earlier.

The erythrocyte membrane lipid order was determined by measuring the fluorescence anisotropy (FAn) of lipid probes inserted into the erythrocyte ghosts. Three fluorescence probes were used, namely 1,6-diphenyl-1,3,5-hexatriene
(DPH), 1-(4-trimethylammonium)-6-phenyl-1,3,5-hexatriene (TMA-DPH) and 8-anilino-1-napthalenesulphonic acid (ANS). DPH and TMA-DPH were dissolved in tetrahydrofuran to a final concentration of $2 \mu \mathrm{M}$ while ANS was dissolved in ethanol to a final concentration of $6 \mu \mathrm{M}^{15}$. The probe stock solutions were diluted (1:1000) in $0 \cdot 155 \mathrm{M}-\mathrm{NaCl}^{14}$ with vigorous mixing before being incubated with erythrocyte ghosts. Working probe solutions were incubated with erythrocyte ghost solution (optical density 0.3 ) at a ratio of $3: 1$ at temperature of $37^{\circ} \mathrm{C}$. The incubation time for DPH and TMA-DPH was $60 \mathrm{~min}$, while that for ANS was $90 \mathrm{~min}$. FAn was measured using a luminescence spectrophotometer (LS-50; Perkin Elmer, Wellesley, MA, USA). Excitation wavelength for DPH and TMA-DPH was $365 \mathrm{~nm}$, while for ANS was $390 \mathrm{~nm}$. Emission was determined at $445 \mathrm{~nm}$ for DPH and TMA-DPH, and $490 \mathrm{~nm}$ for $\mathrm{ANS}^{15}$. An unlabelled erythrocyte ghost was used as a blank. FAn was calculated according to the equation:

$$
A_{\mathrm{r}}=\left(I_{\mathrm{vv}}-G I_{\mathrm{vh}}\right) /\left(I_{\mathrm{vv}}+2 G I_{\mathrm{vh}}\right)
$$

where $I_{\mathrm{vv}}$ and $I_{\mathrm{vh}}$ are the fluroscence intensities obtained from a vertical polarizer, and a vertical and horizontal analyser, while $G$ is the instrumental grating factor. $G=I_{\mathrm{hv}} / I_{\mathrm{vh}}$ where $I_{\mathrm{hv}}$ is the intensity measured from a horizontal polarizer and a vertical analyser ${ }^{15}$.

\section{Statistical analyses}

A repeated measures analysis was used to compare the average means of the four treatment groups in a $2 \times 2$ factorial design (SPSS version 10.0; SPSS Inc., Chicago, IL, USA). The factors used were with or without synbiotic treatment, dietary fat content of $5 \%$ and $15 \%$, and experimental period. ANOVA was used to perform multiple comparisons between means. All data are presented as means and their standard errors $(n 6)$.

\section{Results}

Pigs were healthy in general during the 8 weeks of the experimental period. The growth rate of pigs, and their feed consumption and feed efficiency are shown in Table 2 . The supplementation of synbiotic did not exhibit a significant effect on growth rate $(P=0.225)$, weekly feed intake $(P=0.411)$ and feed efficiency $(P=0 \cdot 474)$. Due to different fat content in the basal diet, feed was adjusted as per energy intake of the pigs; $14.6 \mathrm{MJ} / \mathrm{kg}$ for those on the basal diet containing $5 \%$ fat and $16.9 \mathrm{MJ} / \mathrm{kg}$ for those on the $15 \%$ fat diet. This has contributed to insignificant effect of dietary fat on the growth rate $(P=0.124)$, feed intake $(P=0.456)$ and feed efficiency $(P=0.093)$.

The effect of synbiotic supplementation and dietary fat on lipid profiles is shown in Table 3. The plasma total cholesterol decreased in pigs supplemented with synbiotic on both dietary fats, while those without supplementation increased over 8 weeks $(P=0 \cdot 001)$. Pigs on the high-fat diet also showed higher plasma total cholesterol concentration than those on the low-fat diet $(P=0 \cdot 016)$. However, those given synbiotic and the low-fat diet showed a larger decrease $(18.16 \%)$ than those on the high-fat diet $(1.51 \%)$ over 8 
Table 2. Effect of the supplementation of synbiotic and dietary fat on the growth rate, feed intake and feed efficiency of hypercholesterolaemic pigs (six per group) for 8 weeks*

(Mean values)

\begin{tabular}{|c|c|c|c|c|c|c|c|c|}
\hline \multirow{2}{*}{$\begin{array}{l}\text { Treatment ... } \\
\text { Dietary fat (\%) }\end{array}$} & \multicolumn{2}{|c|}{ Control } & \multicolumn{2}{|c|}{ Synbiotic } & \multirow[b]{2}{*}{ SE† } & \multicolumn{3}{|c|}{$\begin{array}{c}\text { Statistical significance } \\
\text { of effect: } P\end{array}$} \\
\hline & 5 & 15 & 5 & 15 & & $\mathrm{~T}$ & $\mathrm{~F}$ & $T \times F$ \\
\hline Growth rate (kg/pig per week) & 7.98 & 8.50 & $7 \cdot 88$ & 8.05 & 0.22 & 0.225 & 0.124 & 0.433 \\
\hline Feed intake (MJ/pig per week) & $250 \cdot 7$ & $254 \cdot 8$ & $247 \cdot 3$ & $250 \cdot 3$ & 1.54 & 0.411 & 0.456 & 0.911 \\
\hline Growth rate: feed intake & 0.032 & 0.033 & 0.032 & 0.032 & 0.0003 & 0.474 & 0.093 & 0.276 \\
\hline
\end{tabular}

F, effect of dietary fat; $T$, effect of treatment.

* The control diet contained no synbiotic while the synbiotic diet contained Lactobacillus acidophilus ATCC 4962 (1.00 g/pig per d), fructooligosaccharide $(1.25 \mathrm{~g} /$ pig per d), mannitol ( $1.56 \mathrm{~g} /$ pig per d) and inulin $(2.20 \mathrm{~g} /$ pig per $\mathrm{d})$.

t Standard error of the difference for $T \times F$.

†Feed was adjusted weekly according to energy intake per weight of pigs; $14.6 \mathrm{MJ} / \mathrm{kg}$ for those on the basal diet containing $5 \%$ fat and $16.9 \mathrm{MJ} / \mathrm{kg}$ for those on the basal diet containing $15 \%$ fat.

weeks. Pigs on the control diet had an increased fasted plasma TAG of $17.64 \%$ (low-fat diet) and $49.69 \%$ (highfat diet) over 8 weeks. Conversely, those on the synbiotic diet had a decrease of $15.48 \%$ (low-fat diet) and $41.40 \%$ (high-fat diet) $(P=0.002)$. Changes in plasma TAG were more prevalent between treatment groups and dietary fat in non-fasted samples. Pigs given the high-fat diet had significantly higher non-fasted plasma TAG than those on the low-fat diet $(P=0.001)$, while those given the synbiotic had a lower concentration than those without supplementation $(P<0.001)$. The supplementation of synbiotic significantly lowered plasma LDL-cholesterol compared to the control $(P=0.045)$ over 8 weeks; pigs without the supplementation of synbiotic showed an increase of
46.63-59.19\% while pigs given the synbiotic showed an increase of only $13 \cdot 11-28 \cdot 11 \%$. Dietary fat had no effect on the concentration of HDL-cholesterol.

The subfractions of major lipoproteins are shown in Fig. 1. TAG content of VLDL decreased over 8 weeks (Fig. 1(A, D)) for pigs on both dietary treatments and synbiotic supplementation. The low-fat diet had significantly lower concentration of TAG in VLDL than the high-fat diet $(P=0.008)$ over 8 weeks, while the supplementation of synbiotic similarly lowered the concentration of TAG in VLDL over the experimental period compared to pigs without supplementation $(P=0.008)$. This was accompanied by an increasing concentration of $\mathrm{CE}$ in VLDL over the experimental period for all treatment groups.

Table 3. Effect of the supplementation of synbiotic and dietary fat on plasma lipid profiles of hypercholesterolaemic pigs (six per group) for 8 weeks ${ }^{*}$ (Mean values)

\begin{tabular}{|c|c|c|c|c|c|c|c|c|c|c|c|}
\hline & \multirow[b]{2}{*}{ Treatment } & \multirow[b]{2}{*}{ Dietary fat $(\%)$} & \multicolumn{5}{|c|}{ Week } & \multirow[b]{2}{*}{ SE† } & \multicolumn{3}{|c|}{$\begin{array}{l}\text { Statistical significance } \\
\quad \text { of effect: } P\end{array}$} \\
\hline & & & 0 & 2 & 4 & 6 & 8 & & W & $\mathrm{T}$ & $\mathrm{F}$ \\
\hline \multirow[t]{4}{*}{ Total cholesterol $(\mathrm{mmol} / \mathrm{l})$} & \multirow[t]{2}{*}{ Control } & 5 & $6 \cdot 44$ & 4.49 & 6.43 & $7 \cdot 85$ & 8.50 & \multirow[t]{4}{*}{0.23} & \multirow[t]{4}{*}{$<0.001$} & \multirow[t]{4}{*}{0.001} & \multirow[t]{4}{*}{0.016} \\
\hline & & 15 & $6 \cdot 33$ & 5.77 & $6 \cdot 22$ & 8.49 & 8.98 & & & & \\
\hline & \multirow[t]{2}{*}{ Synbiotic } & 5 & 6.93 & 3.86 & $5 \cdot 70$ & 5.97 & $5 \cdot 67$ & & & & \\
\hline & & 15 & 6.92 & 5.48 & 5.07 & $7 \cdot 19$ & $6 \cdot 81$ & & & & \\
\hline \multirow[t]{4}{*}{ TAG (fasted) $(\mathrm{mmol} / \mathrm{l})$} & \multirow[t]{2}{*}{ Control } & 5 & 1.77 & 1.65 & $2 \cdot 23$ & 2.06 & 2.08 & \multirow[t]{4}{*}{0.11} & \multirow[t]{4}{*}{0.090} & \multirow[t]{4}{*}{0.002} & \multirow[t]{4}{*}{0.075} \\
\hline & & 15 & 1.60 & $2 \cdot 34$ & $2 \cdot 35$ & $2 \cdot 29$ & $2 \cdot 40$ & & & & \\
\hline & \multirow[t]{2}{*}{ Synbiotic } & 5 & $2 \cdot 16$ & 1.05 & 1.50 & 1.51 & 1.83 & & & & \\
\hline & & 15 & 2.58 & 1.75 & 1.40 & 1.67 & 1.51 & & & & \\
\hline \multirow[t]{4}{*}{ TAG (non-fasted) (mmol/l) } & \multirow[t]{2}{*}{ Control } & 5 & $3 \cdot 34$ & $5 \cdot 32$ & $4 \cdot 82$ & 4.57 & 4.78 & \multirow[t]{4}{*}{0.40} & \multirow[t]{4}{*}{0.018} & \multirow[t]{4}{*}{$<0.001$} & \multirow[t]{4}{*}{0.001} \\
\hline & & 15 & 3.48 & $7 \cdot 33$ & $6 \cdot 86$ & 7.59 & 7.00 & & & & \\
\hline & \multirow[t]{2}{*}{ Synbiotic } & 5 & 3.52 & 2.54 & $2 \cdot 70$ & $3 \cdot 14$ & 2.59 & & & & \\
\hline & & 15 & $3 \cdot 14$ & $4 \cdot 16$ & $3 \cdot 61$ & 3.38 & $4 \cdot 18$ & & & & \\
\hline \multirow[t]{4}{*}{ LDL-cholesterol (mmol/l) } & \multirow[t]{2}{*}{ Control } & 5 & $4 \cdot 26$ & 5.09 & $5 \cdot 84$ & $6 \cdot 28$ & 6.79 & \multirow[t]{4}{*}{0.25} & \multirow[t]{4}{*}{$<0.001$} & \multirow[t]{4}{*}{0.045} & \multirow[t]{4}{*}{0.859} \\
\hline & & 15 & 4.52 & $5 \cdot 85$ & $6 \cdot 32$ & 6.48 & $6 \cdot 62$ & & & & \\
\hline & Synbiotic & 5 & 4.85 & 5.05 & 5.65 & 5.86 & $6 \cdot 22$ & & & & \\
\hline & & 15 & 4.93 & 5.00 & 5.46 & $5 \cdot 39$ & 5.58 & & & & \\
\hline HDL-cholesterol (mmol/l) & Control & 5 & 0.49 & 0.57 & $0 \cdot 84$ & 0.84 & 0.79 & 0.03 & $<0.001$ & 0.003 & 0.668 \\
\hline & & 15 & 0.55 & 0.68 & 0.83 & 0.83 & 0.99 & & & & \\
\hline & Synbiotic & 5 & 0.67 & 0.73 & 0.77 & 1.04 & 1.06 & & & & \\
\hline & & 15 & 0.69 & 0.63 & 0.69 & 0.95 & 1.07 & & & & \\
\hline
\end{tabular}

$F$, effect of dietary fat; $T$, effect of treatment; $W$, effect of experimental period.

* The control diet contained no synbiotic while the synbiotic diet contained Lactobacillus acidophilus ATCC 4962 ( $1.00 \mathrm{~g} / \mathrm{pig}$ per d), fructooligosaccharide (1.25 g/pig per d), mannitol ( $1.56 \mathrm{~g} /$ pig per d) and inulin $(2.20 \mathrm{~g} /$ pig per d).

† Standard error of the difference for $\mathrm{T} \times \mathrm{F}$. 
(A)

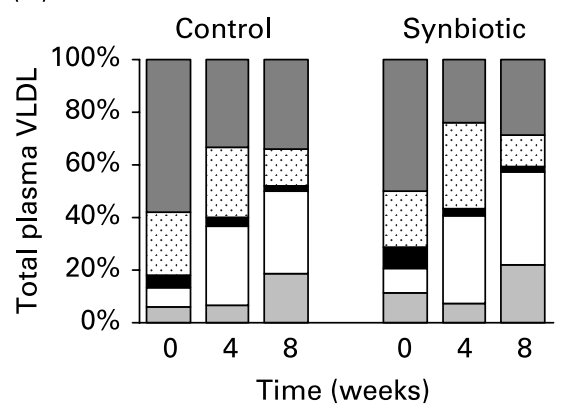

(D)

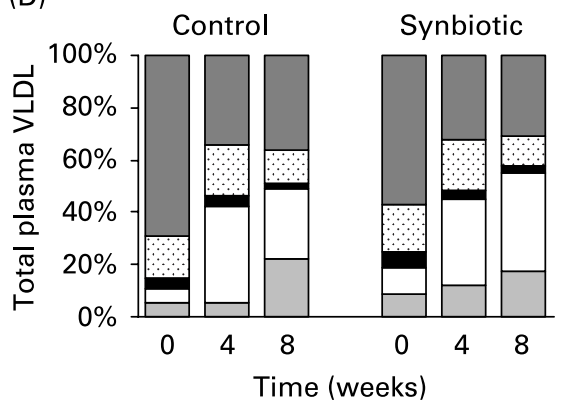

(B)

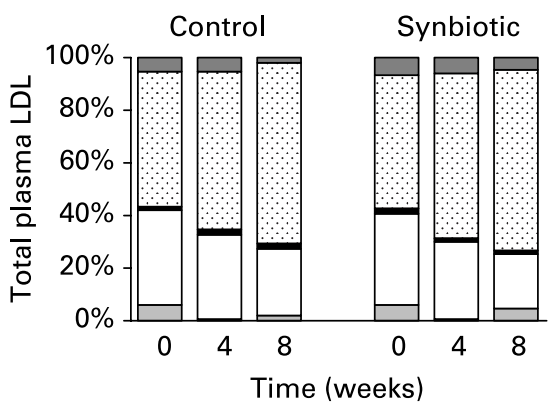

(E)

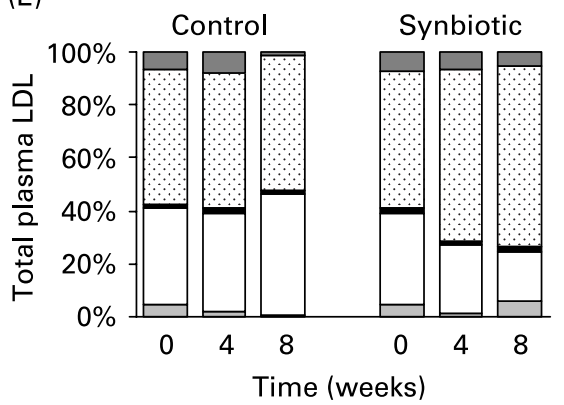

(C)

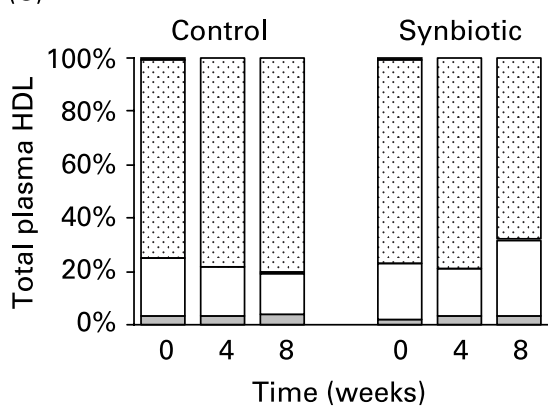

(F)

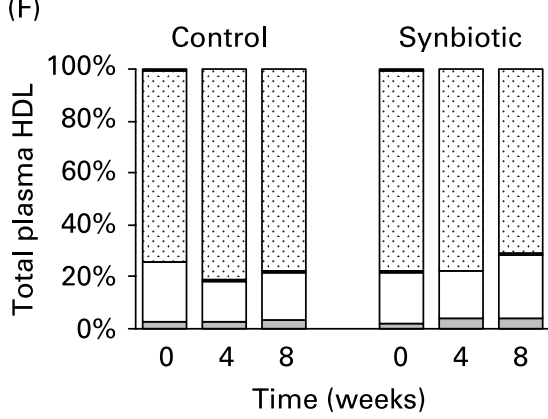

Fig. 1. Subfractions of VLDL-, LDL- and HDL-cholesterol of pigs fed the control and synbiotic diets with $5 \%$ fat $(A, B, C)$ and $15 \%$ fat (D, E, F) for 8 weeks. The control diet contained no synbiotic while the synbiotic diet contained Lactobacillus acidophilus ATCC 4962 ( $1.00 \mathrm{~g} / \mathrm{pig}$ per d), fructooligosaccharide (1.25 g/pig per d), mannitol ( $1.56 \mathrm{~g} /$ pig per d) and inulin ( $2.20 \mathrm{~g} /$ pig per d). Results are expressed as percentage of total plasma lipoproteins: TAG ( $\square$ ), protein ( $\square$ ), phospholipids $(\square)$, cholesteryl esters ( $\square$ ) and free cholesterol (回).

By weight, a large portion of LDL is CE and free cholesterol with little $\mathrm{TAG}^{25}$. This was shown in pigs on all treatments studied (Fig. 1(B, E)). Although the concentration of TAG was small in LDL and was decreasing over 8 weeks for all treatments, pigs supplemented with synbiotic had a significantly smaller decrease $(P=0.025)$ over 8 weeks $(30 \cdot 29-31.65 \%)$ than those without supplementation (63.07-77.58\%), regardless of the dietary fat. However, dietary fat appeared to have no effect on the concentration of TAG in LDL. The supplementation of synbiotic also contributed to a larger decrease $(P<0.001)$ in the concentration of $\mathrm{CE}$ in LDL for both dietary fats (39.36-44.92\%) over 8 weeks than those without supplementation $(25 \cdot 01-30 \cdot 42 \%)$. Pigs on the high-fat diet also had a significantly larger decrease of $\mathrm{CE}$ in LDL than those given the low-fat diet $(P=0.05)$. There was a linear interaction $(P=0.002)$ between experimental period and supplementation of synbiotic such that the concentration of $\mathrm{CE}$ in LDL decreased with time in pigs supplemented with synbiotic while the control remained relatively constant.

The HDL particles contain high amounts of protein (approximately 50\%) with small amounts of TAG (5\%), cholesterol $(20 \%)$ and phospholipids $(25 \%)^{26}$. These have been manifested in pigs on all treatment groups (Fig. 1(C, F)). The concentration of $\mathrm{CE}$ in HDL increased $(P=0.036)$ when pigs were supplemented with synbiotic regardless of dietary fat over the experimental period (25.86-35.09\%), while those without supplementation showed a decrease of $20 \cdot 34-29 \cdot 87 \%$ over 8 weeks.

The morphology of erythrocytes was assessed using Wright's stain and is illustrated in Fig. 2. The erythrocyte morphology appeared to be normal for all treatments initially
(Fig. 2(A-D)), however, at the end of the experimental period, the pigs on the control diet showed a distinct characteristic of spur cells (Fig. 2(E, G)), while the erythrocytes of those on the synbiotic diet were less affected. Further, the occurrence of spur cells was more prevalent in pigs on the high-fat diet (Fig. 2(F)) than those on the low-fat diet (Fig. 2(H)).

Phospholipid content of erythrocyte membranes increased $(P<0.001)$ over the experimental period for all treatments (Table 4). The administration of synbiotic significantly $(P=0.005)$ contributed to higher concentrations of phospholipids compared to that without supplementation. Pigs given the high-fat diet also showed higher concentration of erythrocyte phospholipids than those on the low-fat diet $(P=0.001)$. The diet with higher fat content contributed to higher concentration of cholesterol in the erythrocytes compared to that with lower fat content $(P<0.001)$. There was a significant interaction $(P=0.017)$ between the dietary fat and supplementation of synbiotic; there was a largest increase in the concentration of cholesterol over 8 weeks $(157.67 \%)$ when pigs were not supplemented with synbiotic and were given the high-fat diet, while the concentration of cholesterol in erythrocytes had the smallest increase $(44.58 \%)$ when pigs were supplemented with synbiotic and were given the low-fat diet. The ratio of cholesterol/phospholipids $(\mathrm{C} / \mathrm{P} ; \mathrm{mol} / \mathrm{mol})$ was significantly lower $(P=0.001)$ when pigs consumed synbiotic compared to those that did not. The $\mathrm{C} / \mathrm{P}$ was also lower $(P=0.007)$ when pigs were given the low-fat diet compared to those on the high-fat diet. There was a significant interaction between the experimental period, supplementation of synbiotic and dietary fat $(P=0.004)$ where a smaller increase in $\mathrm{C} / \mathrm{P}$ over 8 weeks was observed in pigs 
(A)

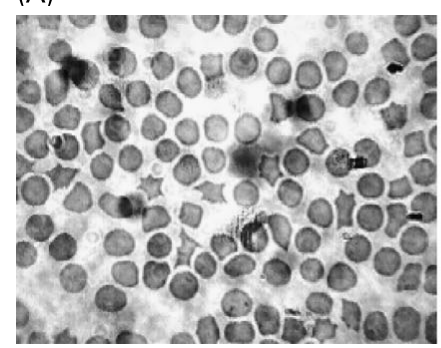

(E)

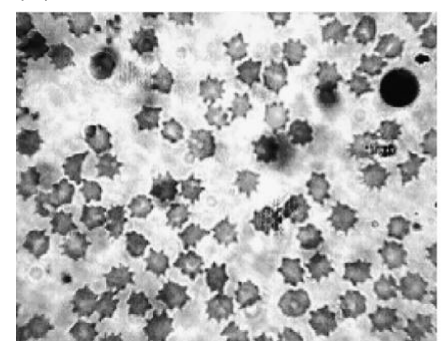

(B)

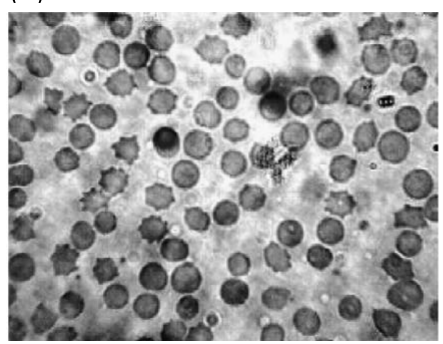

(F)

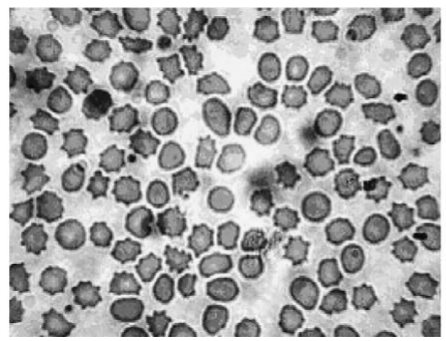

(C)

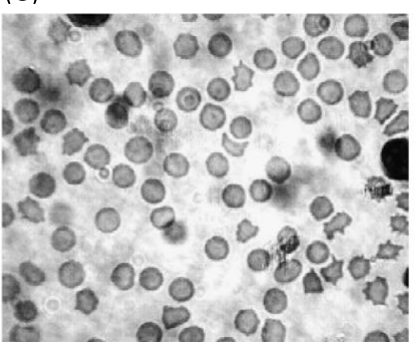

(G)

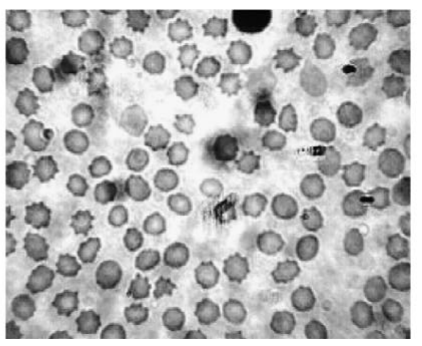

(D)

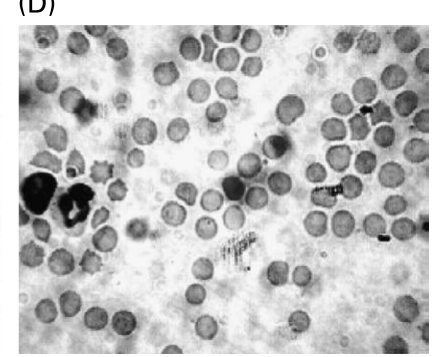

(H)

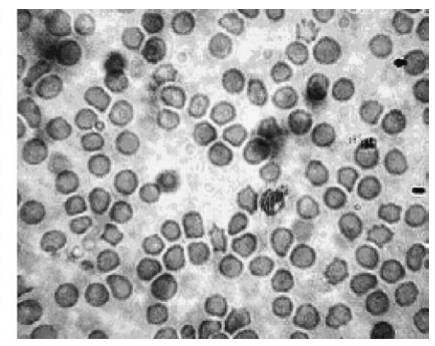

Fig. 2. Morphology of erythrocytes after staining using Wright's stain from pigs $(n 6)$ on the high-fat diet without $(A)$ and with (B) synbiotic at the initial feeding period, pigs on the low-fat diet without (C) and with (D) synbiotic at the initial feeding period, pigs on the high-fat diet without (E) and with (F) synbiotic over 8 weeks, and pigs on the low-fat diet without $(\mathrm{G})$ and with $(\mathrm{H})$ synbiotic over 8 weeks. The synbiotic supplementation contained Lactobacillus acidophilus ATCC $4962(1.00 \mathrm{~g} /$ pig per d), fructooligosaccharide ( $1.25 \mathrm{~g} /$ pig per d), mannitol $(1.56 \mathrm{~g} / \mathrm{pig}$ per d) and inulin $(2.20 \mathrm{~g} / \mathrm{pig}$ per d).

supplemented with synbiotic but was given the high-fat diet $(5.00 \%)$ compared to those given the low-fat diet $(13.58 \%)$.

Considering that the administration of dietary fat affected erythrocyte membrane lipid content and the supplementation of synbiotic could alter such effects, we further evaluated the locations of cholesterol enrichment using fluorescence probes (Table 5). FAn of DPH increased over just 2 weeks for all treatments studied. However, pigs supplemented with synbiotic and given the low-fat diet showed an increase of DPH over 4 weeks $(P<0 \cdot 01)$. The supplementation of synbiotic also reduced the FAn $(P<0.001)$ in pigs for both dietary fat treatments $(10.87-19.57 \%)$ while those without supplementation showed an increase (2.27-39.39\%) over the experimental period. The FAn was also higher $(P=0 \cdot 012)$ when pigs were on the high-fat diet than those on the low-fat diet.

The experimental period significantly affected the FAn of ANS $(P<0.001)$; the low-fat diet contributed to a continuous decrease over 8 weeks regardless of synbiotic supplementation while the high-fat diet contributed to a decrease over $2-4$

Table 4. Effect of the supplementation of synbiotic and dietary fat on concentration of cholesterol and phospholipids in erythrocyte membranes of hypercholesterolaemic pigs (six per group) for 8 weeks*

(Mean values)

\begin{tabular}{|c|c|c|c|c|c|c|c|c|c|c|c|}
\hline & \multirow[b]{2}{*}{ Treatment } & \multirow[b]{2}{*}{ Dietary fat (\%) } & \multicolumn{5}{|c|}{ Week } & \multirow[b]{2}{*}{ SE† } & \multicolumn{3}{|c|}{$\begin{array}{c}\text { Statistical significance of } \\
\text { effect: } P\end{array}$} \\
\hline & & & 0 & 2 & 4 & 6 & 8 & & W & $\mathrm{T}$ & $\mathrm{F}$ \\
\hline \multirow[t]{4}{*}{ Phospholipids ( $\mu \mathrm{g} / 10^{8}$ erythrocytes) } & Control & 5 & 0.44 & 0.66 & 0.70 & 0.66 & 0.65 & 0.03 & $<0.001$ & 0.005 & 0.001 \\
\hline & & 15 & 0.53 & 0.62 & 0.69 & 0.78 & 0.85 & & & & \\
\hline & Synbiotic & 5 & 0.55 & 0.64 & 0.70 & 0.73 & 0.75 & & & & \\
\hline & & 15 & 0.55 & 0.65 & 0.70 & 1.00 & 1.04 & & & & \\
\hline \multirow[t]{4}{*}{ Cholesterol ( $\mu \mathrm{g} / 10^{8}$ erythrocytes) $\ddagger$} & Control & 5 & 3.84 & 6.74 & $7 \cdot 38$ & 7.89 & 8.96 & 0.42 & $<0.001$ & 0.076 & $<0.001$ \\
\hline & & 15 & $5 \cdot 15$ & 11.07 & 11.36 & 11.24 & 13.27 & & & & \\
\hline & Synbiotic & 5 & 5.54 & 7.03 & $6 \cdot 29$ & 8.02 & 8.01 & & & & \\
\hline & & 15 & 5.48 & $7 \cdot 31$ & 7.44 & 11.52 & $10 \cdot 87$ & & & & \\
\hline \multirow[t]{4}{*}{ Cholesterol/phospholipids (mol/mol)§ } & Control & 5 & 0.72 & 0.83 & 0.87 & 0.97 & $1 \cdot 19$ & 0.05 & $<0.001$ & 0.001 & 0.007 \\
\hline & & 15 & 0.78 & 1.47 & 1.35 & 1.17 & 1.30 & & & & \\
\hline & Synbiotic & 5 & 0.81 & 0.88 & 0.73 & 0.88 & 0.92 & & & & \\
\hline & & 15 & 0.80 & 0.92 & 0.86 & 0.93 & 0.84 & & & & \\
\hline
\end{tabular}

$F$, effect of dietary fat; $T$, effect of treatment; $W$, effect of experimental period.

* The control diet contained no synbiotic while the synbiotic diet contained Lactobacillus acidophilus ATCC 4962 ( $1.00 \mathrm{~g} /$ pig per d), fructooligosaccharide (1.25 g/pig per d), mannitol ( $1.56 \mathrm{~g} /$ pig per d) and inulin ( $2.20 \mathrm{~g} /$ pig per d).

$\dagger$ Standard error of the difference for $T \times F$.

$\ddagger \mathrm{T} \times \mathrm{F}$ interaction, $P=0.017$.

$\S \mathrm{W} \times \mathrm{T} \times \mathrm{F}$ interaction, $P=0.004$. 
Table 5. Effect of the supplementation of synbiotic and dietary fat on fluorescence anisotropy of erythrocyte ghosts in hypercholesterolaemic pigs (six per group) for 8 weeks*

(Mean values)

\begin{tabular}{|c|c|c|c|c|c|c|c|c|c|c|c|}
\hline \multirow[b]{2}{*}{ Fluorescence anisotropy } & \multirow[b]{2}{*}{ Treatment } & \multirow[b]{2}{*}{ Dietary fat (\%) } & \multicolumn{5}{|c|}{ Week } & \multirow[b]{2}{*}{ SE† } & \multicolumn{3}{|c|}{$\begin{array}{c}\text { Statistical significance of } \\
\text { effect: } P\end{array}$} \\
\hline & & & 0 & 2 & 4 & 6 & 8 & & W & $\mathrm{T}$ & $\mathrm{F}$ \\
\hline \multirow[t]{4}{*}{$\mathrm{DPH}$} & Control & 5 & 0.44 & 0.68 & 0.51 & 0.41 & 0.45 & 0.02 & $<0.001$ & $<0.001$ & 0.012 \\
\hline & & 15 & 0.33 & 0.84 & 0.54 & 0.4 & 0.46 & & & & \\
\hline & Synbiotic & 5 & 0.46 & 0.56 & 0.68 & 0.29 & 0.37 & & & & \\
\hline & & 15 & 0.46 & 0.71 & 0.45 & 0.33 & 0.41 & & & & \\
\hline \multirow[t]{4}{*}{ ANS } & Control & 5 & 0.43 & 0.42 & 0.27 & 0.24 & 0.25 & 0.01 & $<0.001$ & $<0.001$ & 0.231 \\
\hline & & 15 & 0.3 & 0.46 & 0.25 & 0.27 & 0.23 & & & & \\
\hline & Synbiotic & 5 & 0.41 & 0.41 & 0.37 & 0.3 & 0.26 & & & & \\
\hline & & 15 & 0.34 & 0.40 & 0.44 & 0.27 & 0.25 & & & & \\
\hline \multirow[t]{4}{*}{ TMA-DPH } & Control & 5 & 0.19 & 0.19 & 0.27 & 0.21 & 0.22 & 0.01 & 0.004 & $<0.001$ & 0.040 \\
\hline & & 15 & 0.17 & 0.23 & 0.4 & 0.23 & 0.2 & & & & \\
\hline & Synbiotic & 5 & $0 \cdot 17$ & 0.14 & 0.13 & 0.2 & 0.15 & & & & \\
\hline & & 15 & $0 \cdot 16$ & 0.18 & 0.18 & 0.22 & $0 \cdot 16$ & & & & \\
\hline
\end{tabular}

ANS, 8-anilino-1-napthalenesulphonic acid; DPH, 1,6-diphenyl-1,3,5-hexatriene; F, effect of dietary fat; T, effect of treatment; TMA-DPH, 1-(4-trimethylammonium)-6-phenyl1,3,5-hexatriene; $\mathrm{W}$, effect of experimental period.

${ }^{*}$ The control diet contained no synbiotic while the synbiotic diet contained Lactobacillus acidophilus ATCC 4962 ( $1.00 \mathrm{~g} / \mathrm{pig}$ per d), fructooligosaccharide (1.25 g/pig per d), mannitol ( $1.56 \mathrm{~g} /$ pig per d) and inulin ( $2.20 \mathrm{~g} /$ pig per d).

† Standard error of the difference for $\mathrm{T} \times \mathrm{F}$.

weeks. Also, pigs supplemented with synbiotic required a longer period before reducing the FAn of ANS compared to those without supplementation, accompanied by a smaller decrease of $26.47-36.59 \%$ over 8 weeks while those without supplementation decreased by $23 \cdot 33-41 \cdot 86 \%(P<0 \cdot 001)$.

There were significant changes $(P=0.004)$ in the FAn of TMA-DPH over the experimental period; pigs without the supplementation of synbiotic showed reduced FAn of TMADPH over 4 weeks for both the high- and low-fat diets, while the FAn for pigs supplemented with synbiotic only reduced over 6 weeks. The administration of synbiotic also reduced the FAn in pigs on both dietary fat treatments compared to those that did not consume the synbiotic $(P<0.001)$, accompanied by a lower increase over 8 weeks $(0.00-11.76 \%)$ compared to an increase of $15.79-17.65 \%$ when pigs were not given the synbiotic. The high-fat diet also significantly contributed to a higher FAn $(P=0 \cdot 040)$ of TMA-DPH than the low-fat diet.

\section{Discussion}

It has been previously found that modifications in dietary fats and fatty acids may affect plasma lipoprotein profiles ${ }^{27}$. However, the present study indicated that only plasma total cholesterol and TAG (non-fasted) concentrations were significantly affected $(P=0.016$ and $P=0.001$, respectively) by the different diets while the other lipoprotein parameters were not affected. Although the high-fat diet had higher fat content, higher SFA and lower PUFA concentrations than the low-fat diet, these differences also had insignificant effect on the growth rate $(P=0 \cdot 124)$, feed intake $(P=0.456)$ and feed efficiency $(P=0.093)$ in all pigs.

The current study showed that the supplementation of synbiotic reduced plasma TAG (fasted and non-fasted), total cholesterol and LDL-cholesterol in pigs. Hypercholesterolaemia has been associated with higher than normal total cholesterol $(6.21 \mathrm{mmol} / \mathrm{l})$ concentration. Although pigs given the high-fat diet remained hypercholesterolaemic over the experimental period, the administration of synbiotic contributed to a decreased plasma cholesterol concentration. When the low-fat diet was used, pigs without the supplementation of synbiotic remained hypocholesterolaemic over 8 weeks, while those given the synbiotic showed a reduction to normal concentration over 8 weeks. Higher than normal concentration of LDL-cholesterol $(4.41 \mathrm{mmol} / \mathrm{l})$ is also an attribute to hypercholesterolaemia. Although the supplementation of synbiotic improved the LDL-cholesterol content, it failed to achieve a normal concentration in all pigs.

There is very little information on the source and nature of abnormalities in lipoproteins ${ }^{11,12}$. Hence, we wanted to evaluate further the compositions of these lipoprotein classes in order to better understand the alteration of lipid components as affected by the synbiotic product that was developed in our previous study ${ }^{16}$.

VLDL is primarily synthesized in the liver and contains about $50-60 \%$ of TAG, which is the major lipid class that is transported from the liver into the bloodstream ${ }^{28}$. Results from the present study showed that pigs on all dietary treatments had normal concentrations of TAG in the subfraction of VLDL at the initial point of the feeding trial, but were reduced over 8 weeks. VLDL particles are the precursors for LDL particles where the TAG in VLDL are exchanged for $\mathrm{CE}$ in the core of $\mathrm{LDL}^{13}$. Results from the present study showed that pigs supplemented with the synbiotic decreased CE and increased TAG in LDL, complemented by a lower concentration of TAG in VLDL, indicating higher conversion of VLDL into LDL.

In addition, synbiotic also beneficially decreased the concentration of $\mathrm{CE}$ in LDL. Higher concentration of $\mathrm{CE}$ in LDL induced by diet was found to be associated with an increased risk for atherosclerosis in African green monkeys ${ }^{29}$. More importantly, such TAG-enriched LDL particles are more susceptible to further hydrolysis ${ }^{28}$ and are removed from blood via binding to LDL receptors, where the CE particles 
are hydrolysed into free cholesterol ${ }^{30}$. Loss of CE from the core of LDL forms smaller and denser LDL particles. Although smaller LDL appeared more atherogenic than larger LDL particles ${ }^{31}$, smaller LDL formed as a result of dietary intervention was removed by plasma more rapidly than larger particles ${ }^{32}$. Results from the present study clearly showed that the administration of the synbiotic decreased the concentration of $\mathrm{CE}$ coupled with increased concentration of TAG in the LDL subfraction, which may have contributed to the improved plasma LDL-cholesterol in pigs supplemented with synbiotic.

The major function of HDL is to transport cholesterol back to the liver, and this could involve a process referred to as reverse cholesterol transport ${ }^{33}$. Briefly, free cholesterol is transported into HDL particles and subsequently esterified to form CE. HDL contains mainly lecithin and protein, with small amount of cholesterol and TAG. Thus, matured HDL with bigger core containing more $\mathrm{CE}$ are transported back to the liver and hydrolysed. This CE is transferred by CE transfer protein and internalized by the LDL receptor. The HDL is then released for further reverse cholesterol transport ${ }^{28}$. Experimental data from the present study showed that the administration of synbiotic increased the concentration of $\mathrm{CE}$ in the HDL subfraction, which indicated that cholesterol was removed by HDL in the form of CE. This explains why there was reduced plasma total cholesterol in pigs supplemented with synbiotic.

Although results from the present study showed increased concentration of $\mathrm{CE}$ in the HDL particles, pigs supplemented with synbiotic also had lower plasma HDLcholesterol than those without supplementation. Low plasma HDL-cholesterol is not an indicator of faulty removal of cholesterol through deficient activity of the reverse cholesterol transport pathway ${ }^{28}$. Instead, the $\mathrm{CE}$ in the core of HDL may also be exchanged by CE transfer protein for the TAG in VLDL, producing a TAG-enriched but CE-depleted HDL, which appeared to be catabolized more rapidly by the kidney. The HDL-cholesterol concentration is thus decreased ${ }^{13}$. Results from the present study showed that using the direct concentration of plasma HDL-cholesterol as an indicator for healthy lipoprotein profiles would be misleading, as a higher concentration of plasma HDL-cholesterol does not indicate high efficiency in cholesterol transport.

The occurrence of spur cells appeared to be improved by the supplementation of synbiotic as supported by the morphological representation and lower $\mathrm{C} / \mathrm{P}$ ratio. Cholesterol in the membrane lipid bilayer prevents fatty acid chains from coming together and crystallizing; however, cholesterol also decreases the permeability of the bilayer to small water-soluble molecules, thus reducing fluidity ${ }^{34}$. It has been reported that the normal $\mathrm{C} / \mathrm{P}$ ratio of erythrocytes for dogs is approximately $1 \cdot 05^{24}$. At the end of the experimental period, $\mathrm{C} / \mathrm{P}$ ratio for the pigs with the supplementation of synbiotic was normal $(0.84-0.92)$ while those without supplementation were higher than the normal ratio $(1 \cdot 19-1 \cdot 30)$. Such abnormality in lipid composition would result in an increase in the surface area of the cells, thus increasing rigidity and decreasing fluidity. These led to a decreased ability of the cell to deform and thus the occurrence of spur cells ${ }^{18}$.
Although the present results and previous studies ${ }^{14}$ showed that dietary fat intervention contributed to the enrichment of cholesterol in erythrocyte membranes, we were unable to distinguish the location of such enrichment in the membranes. Thus, we have used three different fluorescence probes to allow examination in different locations of the membrane bilayer. These probes exhibit strong fluorescence increase upon binding to lipids and have sensitive anisotropy responses to lipid order. DPH is an apolar fluorophore mainly incorporated into different apolar regions of the membrane ${ }^{35}$. The experimental data showed that pigs supplemented with the synbiotic had lower saturation of cholesterol within the apolar region of the erythrocytes. A higher FAn of $\mathrm{DPH}$ in pigs without synbiotic indicated enrichment of cholesterol in the region close to the acyl chains of the phospholipid tails ${ }^{36}$.

ANS has high affinity towards the interface between the apolar tail and the polar head of phospholipids, indicating changes in the surface ${ }^{15}$, and has been associated with the insertion of positively charged molecules into the bilayer, thus increasing the packing order of the surface and the inner hydrocarbon region ${ }^{37}$. The supplementation of synbiotic had little effect when pigs were given the low-fat diet. However, when pigs were on the high-fat diet, the supplementation of synbiotic had a reduced and delayed increase of cholesterol saturation in the upper phospholipids region, indicating improved membrane fluidity via increased permeability and decreased packing order.

TMA-DPH is a cationic probe with the fluorophore TMA incorporated in the head group and DPH incorporated in the acyl chain ${ }^{38}$, and been associated with reduced water penetrability into the bilayer, more ordered bilayer structure and higher cholesterol concentrations in the head group ${ }^{39}$. Results from the present study showed that the supplementation of synbiotic reduced the packing order and saturation of cholesterol in the polar heads of the membrane bilayer.

In conclusion, the supplementation of synbiotic reduced plasma TAG, total cholesterol and LDL-cholesterol in hypercholesterolaemic pigs. Evaluation of compositions of individual lipoprotein classes suggested that the synbiotic may reduce cholesterol in the form of $\mathrm{CE}$ via the interrelated pathways of lipid transporters (VLDL, LDL and HDL). The synbiotic also appeared to improve the occurrence of spur cells. The present study provided the experimental evidence that the supplementation of synbiotic reduced cholesterol saturation within the apolar tails of phospholipids, within the interfacial regions of the apolar tail and polar heads, and improved penetrability of the head group.

\section{References}

1. Reid G, Jass J, Sebulsky MT \& McCormick JK (2003) Potential uses of probiotics in clinical practice. Clin Microbiol Rev 4, 658-672.

2. Hamilton-Miller JM (2004) Probiotics and prebiotics for the elderly. Postgrad Med J 80, 447-451.

3. Flickinger EA, Wolf BW \& Garleb KA (2000) Glucose-based oligosaccharides exhibit different in vitro fermentation patterns and affect in vivo apparent nutrient digestibility and microbial populations in dogs. J Nutr 130, 1267-1273. 
4. Gibson GR \& Roberfroid MB (1995) Dietary modulation of the human colonic microbiota: introducing the concept of prebiotics. J Nutr 125, 1401-1412.

5. Kenney J (2002) Low-fat diets more effective than high-fat diets for both weight loss and health. Pritikin Perspec 11, 1-8.

6. Chyou PH \& Eaker ED (2000) Serum cholesterol concentrations and all-cause mortality in older people. Age Aging 29, 69-74.

7. Liong MT \& Shah NP (2005) Acid and bile tolerance and cholesterol removal ability of lactobacilli strains. J Dairy Sci 88, 55-66.

8. Liong MT \& Shah NP (2005) Bile salt deconjugation and BSH activity of five bifidobacterial strains and their cholesterol co-precipitating properties. Food Res Int 38, 135-142.

9. Marlett JA (1997) Sites and mechanisms for the hypercholesterolemic actions of soluble dietary fibre sources. Adv Exp Med Biol 427, 109-121.

10. Hara H, Li S, Sasaki M, et al. (1994) Effective dose of lactosucrose on faecal flora and faecal metabolites of humans. Biosci Microflora 13, 51-63.

11. Jin LZ, Ho YW, Abdullah N \& Jalaludin S (1998) Growth performance, intestinal microbial populations and serum cholesterol of broiler on diets containing Lactobacillus culture. Poult Sci 77, 1259-1265.

12. Lin SY, Ayres JW, Winkler W \& Sandine WE (1989) Lactobacillus effects on cholesterol: in vitro and in vivo results. J Dairy Res 72, 2885-2899.

13. Kwiterovich DO Jr (2000) The metabolic pathways of high-density lipoprotein, low-density lipoprotein, and triacylglycerol: a current review. Am J Cardiol 86, 5L-10L.

14. Cooper RA, Leslie MH, Knight D \& Detweiler K (1980) Red blood cholesterol enrichment and spur cell anemia in dogs fed a cholesterol-enriched, atherogenic diet. J Lipid Res 21, 1082-1089.

15. Barshtein G, Bergelson L, Dagan A, Gratton E \& Yedgar S (1997) Membrane lipid order of human red blood cells is altered by physiological levels of hydrostatic pressure. Am J Physiol 272, H538-H543.

16. Liong MT \& Shah NP (2005) Optimization of cholesterol removal, growth and fermentation patterns of Lactobacillus acidophilus ATCC 4962 in presence of mannitol, FOS and inulin: a response surface methodology approach. J Appl Microbiol 98, 1115-1126.

17. Chapman MJ, Goldstein S, Lagrange D \& Lapaud PM (1981) A density gradient ultracentrifugal procedure for isolation of the major lipoprotein classes from human serum. $J$ Lipid Res 22, 339-358.

18. Duhamel G, Forgez P, Nalpas B, Berthelot P \& Chapman MJ (1983) Spur cells in patients with alcoholic liver cirrhosis are associated with reduced plasma levels of apoA-II, $\mathrm{HDL}_{3}$, and LDL. J Lipid Res 24, 1612-1625.

19. Dumaswala UJ, Dumaswala RU, Levin DS \& Greenwalt TJ (1996) Improved red blood cell preservation correlates with decreased loss of bands $3,4 \cdot 1$, acetylcholinerase and lipids in microvesicles. Blood 4, 1612-1616.

20. Dhaliwal BS \& Steinbrecher UP (2000) Cholesterol delivered to macrophages by oxidized low density lipoprotein is sequestered in lysosomes and fails to efflux normally. J Lipid Res 41, $1658-1665$.

21. Lowry OH, Rosebrough NJ, Farr AL \& Randall RJ (1951) Protein measurement with the Folin phenol reagent. $J$ Biol Chem 193, 265-275.
22. Sardet C, Hansma H \& Ostwald R (1972) Characterization of guinea pig plasma lipoproteins: the appearance of new lipoproteins in response to dietary cholesterol. J Lipid Res 13, 624-639.

23. Daly JA \& Ertingshausen G (1972) Direct method for determining inorganic phosphate in serum with the 'Centrifichem'. Clin Chem 18, 263-265.

24. Cooper RA, Leslie MH, Fischkoff S, Shinitzky M \& Shattil SJ (1978) Factors influencing the lipid composition and fluidity of red cell membranes in vitro: production of red cells possessing more than two cholesterols per phospholipid. Biochemistry 17, $327-331$.

25. McEneny J, McMaster C, Trimble ER \& Young IS (2002) Rapid isolation of VLDL subfractions: assessment of composition and susceptibility to copper-mediated oxidation. J Lipid Res 43, 824-831.

26. Sanchez-Muniz FJ, Merinero MC, Rodríguez-Gil S, Ordovas JM, Rodenas S \& Cuesta C (2002) Dietary fat saturation affects apolipoprotein AII levels and HDL composition in postmenopausal women. $J$ Nutr 132, 50-54.

27. Tholstrup T, Sandstrom B, Bysted A \& Holmer G (2001) Effect of 6 dietary fatty acids on the postprandial lipid profile, plasma fatty acids, lipoprotein lipase, and cholesterol ester transfer activities in healthy young men. Am J Clin Nutr 73, 198-208.

28. Kwiterovich DO Jr (2002) Lipoprotein heterogeneity: diagnostic and therapeutic implications. Am J Cardiol 90, 1i-10i.

29. Carr TP, Parks JS \& Rudel LL (1992) Hepatic ACAT activity in African green monkeys is highly correlated to plasma LDL cholesteryl ester enrichment and coronary artery atherosclerosis. Arterioscler Thromb Vasc Biol 12, 1274-1283.

30. Horton JD, Goldstein JL \& Brown MS (2002) SREBPs: activators of the complete program of cholesterol and fatty acid synthesis in the liver. J Clin Invest 109, 1125-1131.

31. Haffner SM (2002) Lipoprotein disorders associated with type-2 diabetes and insulin resistance. Am J Cardiol 90, 55i-61i.

32. Fernandez ML, Abdel-Fattah G \& McNamara DJ (1993) Dietary fat saturation modifies the metabolism of LDL sub-fractions in guinea pigs. Arterioscler Thromb Vasc Biol 13, 1418-1428.

33. Rader DJ (2002) High density lipoproteins and atherosclerosis. Am J Cardiol 90, 62i-70i.

34. Simons K \& Ehehalt R (2002) Cholesterol, lipid rafts and disease. J Clin Invest 110, 597-603.

35. Kaiser RD \& London E (1998) Location of duphenylhexatriene (DPH) and its derivatives within membranes: comparison of different fluorescence quenching analyses of membrane depth. Biochemistry 37, 8180-8190.

36. Roy S, Mohanty A \& Dey J (2005) Microviscosity of bilayer membranes of some $\mathrm{N}$-acylamino acid surfactants determined by fluorescence probe method. Chem Phys Lett 414, 23-27.

37. Sujatha J \& Mishra AK (1997) Effect of ionic and neutral surfactants on the properties of phospholipid vesicles: investigation using fluorescence probes. J Photochem Photobiol A: Chem 104, 173-178.

38. Ben-Yashar V \& Barenholz Y (1991) Characterization of the core and surface of human plasma lipoproteins. A study based on the use of five fluorophores. Chem Phys Lipids 60, 1-14.

39. Straume M \& Litman BJ (1988) Equilibrium and dynamic bilayer structural properties of unsaturated acyl chain phosphatidylcholine-cholesterol-rhodopsin recombinant vesicles and rod outer segment disk membranes as determined from higher order analysis of fluorescence anisotropy decay. Biochemistry 27, $7723-7733$. 